
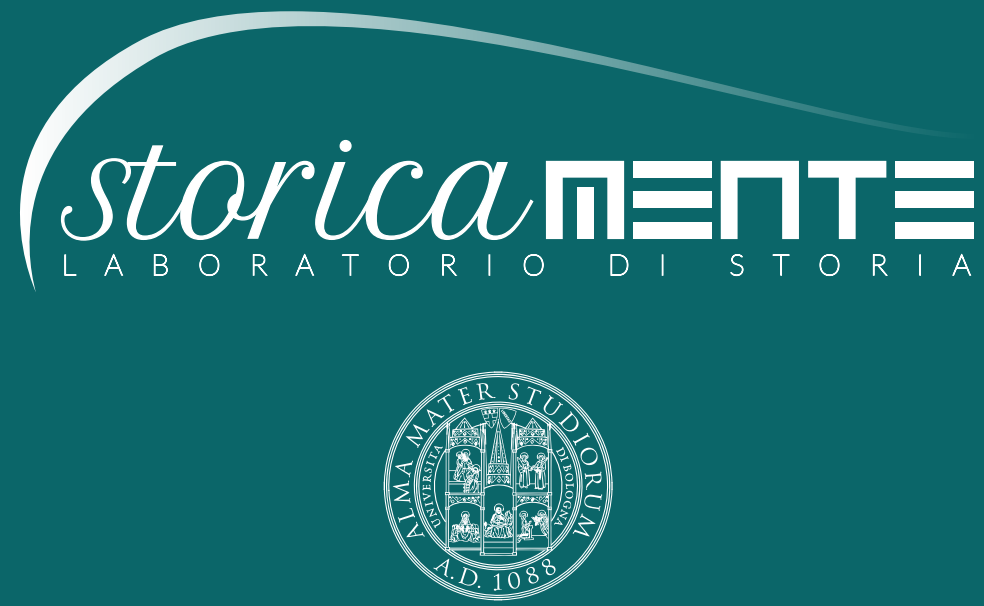

ALMA MATER STUDIORUM

Università di Bologna

Dipartimento di Storia Culture Civiltà 


\section{STORICAMENTE.ORG}

\section{Laboratorio di Storia}

\section{Paolo Capuzzo}

Le nuove generazioni nella crisi italiana:

alcuni contributi sociologici

Numero 10 - 2014

ISSN: $1825-411 \mathrm{X}$

Art. 9

pp. 1-13

DOI: $10.12977 /$ stor515

Editore: BraDypUS

Data di pubblicazione: 14/06/2014

Sezione: Dibattiti 


\title{
Le nuove generazioni nella crisi italiana: alcuni contributi sociologici
}

\author{
PAOLO CAPUZZO \\ Univ. Bologna, \\ Dipartimento di Storia Culture Civiltà
}

L'articolo analizza alcune ricerche comparative sulle differenti "esperienze generazionali" in Italia tra il miracolo economico e l'attuale crisi, evidenziando l'utilità e i limiti della categoria storica di "generazione". Mentre la categoria di generazione rappresenta un utile punto di vista da cui osservare i cambiamenti sociali all'interno di un contesto ampio pervaso da molteplici forze sociali, diviene invece un concetto piuttosto stretto e fuorviante se considerato come una fonte isolata di identità sociale.

This contribute discusses some comparative researches on the different "generational experiences" in Italy between the economic miracle and the current crisis, shedding light on the usefulness and the limits of "generation" as a historical category. While generation represents a useful point of view from which to look at the social change within a wide context pervaded by multiple social forces, it becomes a rather narrow and misleading concept when considered as an isolated source of social identity.

Il fatto che l'appartenenza a una generazione condizioni in modo specifico il rapporto che si ha con il proprio presente storico fa sì che la struttura temporale di una società sia estremamente complessa, essendo dipendente dalla diversità delle esperienze generazionali che in essa convivono. Possiamo perciò utilmente pensare alle generazioni come a un'articolazione della società piuttosto che come a una serie di ondate che continuamente si frangono sui lidi della storia per rifluire poi nell'indistinto. Oltretutto, dal punto di vista biologico, l'appartenenza genera- 
zionale è oggettiva e perciò non revocabile con una scelta soggettiva. Ciò non comporta automaticamente delle conseguenze apprezzabili sul piano storico-sociale, come ha chiarito Mannheim, ma rappresenta una potenzialità che può poi dispiegarsi o meno in relazione ad altre forze che agiscono per la trasformazione della società [Mannheim 2008].

Lo studio dell'avvicendarsi delle generazioni fornisce dunque uno specifico punto di osservazione sulla complessa articolazione delle forze che contribuiscono al cambiamento sociale. L'affacciarsi sulla scena storica di nuove generazioni di giovani nell'Europa del Novecento è stato scandito dalle tragedie della prima metà del secolo. Le due guerre mondiali hanno infatti incorniciato una guerra civile europea che ha visto i giovani come protagonisti: combattenti e animatori delle nuove culture politiche che hanno segnato il secolo, in primo luogo il comunismo e il fascismo. Nella seconda metà del Novecento le giovani generazioni si sono messe in evidenza in contesti fortunatamente meno violenti, ma nondimeno, attraverso nuovi stili di vita e pratiche di consumo, hanno marcato una precisa fase di cambiamento storico-sociale iniziata negli anni Cinquanta e culminata nel 1968: una grande rivolta globale che ha assunto un evidente carattere generazionale.

Accanto ai grandi tornanti della storia del Ventesimo secolo, che hanno permesso l'identificazione generazionale su un piano globale, vi sono poi eventi che hanno assunto un carattere locale. In Italia possiamo ad esempio parlare della generazione del ' 77 o di quella della Pantera, mentre la partecipazione alla fase di alta mobilitazione del movimento alterglobalista tra Seattle e Genova ha assunto dei tratti generazionali più deboli, dato il virtuoso carattere intergenerazionale che ha arricchito il profilo del vasto movimento di opposizione sociale alla globalizzazione neoliberista.

Si ha l'impressione che a partire dagli anni Ottanta sia diventato più difficile identificare dei movimenti giovanili in termini netti, nei quali coincidano il dato biologico della giovinezza e una caratterizzazio- 
ne culturale specifica ed esclusiva [Capuzzo 2012]. La dinamica delle subculture, infatti, se da un lato frammenta i gruppi giovanili secondo orientamenti sempre più diversificati, dall'altro forma delle aggregazioni che si identificano per affinità di valori, comportamenti, gusti e scelte estetiche, con una crescente permeabilità tra generazioni diverse. Inoltre, determinati riti di passaggio hanno sbiadito la loro forza simbolica e detengono un potere di regolazione sociale meno condizionante che nel passato, rendendo più labile il confine tra la fase della giovinezza e l'età adulta. Nella comunicazione giornalistica questi cambiamenti vengono spesso rappresentati nei termini di un soggettivo rifiuto di assunzione delle responsabilità legate alla vita adulta, un fenomeno per il quale la psicologia da rotocalco ha coniato l'espressione di "sindrome di Peter Pan". In verità credo che si tratti di un fenomeno più complesso, nel quale sono certamente implicati degli aspetti psicologici che vanno tuttavia considerati, come si vedrà, nel quadro di più ampi mutamenti storico-sociali e tenendo ben presente la trasformazione di alcuni caratteri biologici del genere umano intervenuta a partire dalla seconda metà del Novecento, in primo luogo il significativo aumento dell'aspettativa di vita. Questi processi ci porteranno probabilmente oltre le modalità del conflitto generazionale che ha caratterizzato il secolo passato e che ha rappresentato uno degli elementi dinamici che hanno segnato i grandi tornanti della storia del Novecento.

Nella prospettiva di un ripensamento della categoria di generazione per la ricerca storica, i testi qui discussi, di impianto prevalentemente sociologico, mi sembra possano offrire degli interessanti spunti di riflessione. Anzitutto, il carattere quantitativo di queste ricerche, che presuppongono una definizione di tipo "oggettivo" dell'appartenenza generazionale, fornisce un solido fondamento allo studio della società secondo coorti di età, particolarmente importante in un momento in cui l'identificazione storico-culturale delle giovani generazioni appare sempre più incerta. Ciò consente di allargare l'approccio allo studio dei giovani oltre la vulgata degli studi culturali, che dopo aver dato un contributo di inno- 
vazione fondamentale allo studio di questi temi negli anni Settanta e Ottanta, hanno visto spesso restringersi la loro capacità di elaborazione critica all'interno di un reificato concetto di subcultura. Del resto, ancor più fuorvianti appaiono gli approcci alla storia dei giovani che considerano questi ultimi soltanto nel quadro dei movimenti politici più o meno organizzati, facendone l'epitome della condizione giovanile tout court.

Lo sguardo sui giovani da un punto di vista demografico e statistico consente inoltre, ed è questa un'ulteriore fertile chiave di lettura dei processi storici, di analizzare i rapporti tra le generazioni in contesti sociali determinati, evidenziando le condizioni biologiche, economiche, sociali che li strutturano e al contempo indagando i rapporti di potere e le implicazioni psicologiche e soggettive assunti dalle relazioni intergenerazionali. Ciò comporta uno spostamento di focus dallo studio delle generazioni che si risolveva nella "storia dei giovani” alle relazioni tra i diversi gruppi generazionali in virtù delle quali diviene necessario indagare anche la condizione degli anziani, una categoria sociale che, nei paesi occidentali e soprattutto in Italia, ha marcato con la propria presenza un profondo mutamento della società a partire dalla fine del Novecento.

Il volume di Antonio Schizzerotto, Ugo Trivellato e Nicola Sartor (a cura di), Generazioni disuguali. Le condizioni di vita dei giovani di ieri e di oggi: un confronto, Bologna, Il Mulino, 2011, presenta un'ampia ricerca diacronica sulla condizione giovanile nell'Italia di oggi comparata a quella delle giovani generazioni della seconda metà del Novecento; vediamone i principali risultati.

I giovani di oggi hanno una qualificazione scolastica molto superiore a quella delle generazioni che li hanno preceduti, in particolare le giovani donne che, a partire dalla coorte delle nate negli anni Sessanta, hanno invertito uno storico gap di genere. Anche la differenziazione dei percorsi che ha visto tradizionalmente le donne più presenti negli studi umanistico-sociali e gli uomini in quelli tecnico-scientifici, pur non 
essendo del tutto scomparsa, si sta attenuando. L'influenza della classe sociale di appartenenza sulla performance scolastica continua ad avere una certa influenza, ma in misura inferiore a quanto accadeva in passato. Se da questo punto di vista una pluridecennale mobilitazione democratica, che ha teso a rendere meno sessista e classista il sistema educativo del nostro paese, sembra avere conseguito un parziale successo, vi sono tuttavia delle ombre pesanti che spengono l'ottimismo generato da questi dati: tutte le rilevazioni comparative dei risultati raggiunti dalla formazione scolastica danno il nostro paese in drammatico regresso e in peggioramento rispetto al livello degli altri paesi; il peso di diplomi e lauree come valore aggiunto sul mercato del lavoro non sembra essere mai stato così basso. Ciò comporta conseguenze importanti perché, se da un lato il declino qualitativo rischia di svuotare di significato il prolungamento delle carriere scolastiche, dall'altro l'effetto egualitario che l'istruzione potrebbe avere come veicolo di mobilità sociale sembra fortemente minato dalla svalutazione del titolo di studio come elemento di forza nel mercato del lavoro.

Dal punto di vista del lavoro, il tasso di attività dei giovani è aumentato fino al 2007, anno di ultima rilevazione della ricerca qui presentata, la quale non documenta perciò la più recente fase di drammatica crisi degli ultimi anni. I tassi di disoccupazione giovanile sono calati fino al 2008, grazie essenzialmente all'incremento del tasso di occupazione femminile. Vi sono tuttavia anche qui delle note dolenti: sono infatti aumentati i tassi di disoccupazione cumulata nella carriera lavorativa a seguito dell'instabilità dei rapporti di lavoro, un dato che appare più attenuato per le donne solo perché anche in precedenza erano esposte a una forte instabilità occupazionale. Su questo piano lo svantaggio delle giovani generazioni è molto forte e provocato dall'introduzione dei lavori atipici tra gli anni Novanta e Duemila. Se inizialmente queste nuove forme contrattuali hanno evidenziato una serie di problematicità che sembravano poter essere riassorbite dal virtuoso effetto sul mercato che erano ritenute poter generare, a partire dal 2008 hanno fatto esplodere 
un problema drammatico in termini di impatto generazionale della crisi. Il rapporto tra la disoccupazione giovanile e quella generale, che si era costantemente ridotto tra il 1987 e il 2007, è tornato rapidamente a crescere, mentre sul piano delle retribuzioni è terminato un ciclo virtuoso che ha visto crescere i salari di ingresso nel dopoguerra: con i nati negli anni Settanta, infatti, esso ha iniziato a regredire.

Le difficoltà occupazionali ed economiche dei giovani italiani più che dal welfare statale sono compensate dalle famiglie. I giovani di oggi possono infatti contare su un sostegno familiare molto più solido, sia dal punto di vista del reddito che da quello patrimoniale, rispetto alle generazioni precedenti. È evidente tuttavia che ciò non è privo di conseguenze: sul piano sociale ciò comporta un rafforzamento del potere delle generazioni anziane su quelle giovani, visto che queste ultime si trovano spesso in condizione di dipendenza dalle prime. Si tratta inoltre di una tendenza che perpetua le differenze economiche come dimostra il fatto che in Italia il reddito delle famiglie di appartenenza influenza quello dei giovani molto di più di quanto accade negli altri paesi dell'Europa continentale. A questi processi che minano la mobilità sociale intergenerazionale, premiando le posizioni acquisite, si aggiungono effetti deleteri dal punto di vista psicologico, dato che molti giovani si trovano a conseguire tardi una piena autonomia personale. È perciò comprensibile che in queste condizioni vengano spostati in avanti tutti i momenti di passaggio a un'indipendente vita adulta: il primo impiego, la prima unione coniugale, la nascita del primo figlio avvengono infatti molto più tardi che per le precedenti generazioni.

Il declino economico complessivo dell'Italia non è certo estraneo alla difficile condizione economica dei giovani. I processi di globalizzazione, con l'ingresso della Cina e dei paesi dell'Est nel mercato internazionale, ha inasprito la concorrenza, in particolare nei settori industriali labour intensive. Si è assistito così al declino di interi settori industriali che avevano costruito la fortuna dell'Italia negli anni del boom e poi nel, pur effimero, rilancio economico degli anni Ottanta. La dislocazione a 
livello europeo della sovranità monetaria ha fatto anche venir meno uno strumento del quale l'Italia si era spesso servita nei momenti critici, vale a dire l'utilizzo della flessibilità dei cambi per delle svalutazioni competitive che permettevano di conservare il potenziale produttivo orientato al mercato internazionale.

Tuttavia queste condizioni, peraltro variamente condivise anche da altri paesi europei, non sono sufficienti a dar conto della profonda iniquità intergenerazionale che è peculiare del nostro paese. Essa infatti discende da precise scelte politiche, in particolare in ambito pensionistico e del lavoro. Con il passaggio dalla riforma Amato alla riforma Dini del 1995 sono stati esclusi dal vincolo contributivo tutti coloro che a quella data avevano almeno 18 anni di anzianità contributiva. Ciò ha caricato di un fardello economico le generazioni successive. Sul piano del lavoro, poi, se il venir meno della scala mobile negli anni Ottanta ha comportato una riduzione del reddito del lavoro dipendente nel suo complesso, i nuovi contratti atipici introdotti tra il 1997 e il 2003 hanno consentito un'ulteriore compressione dei salari che ha riguardato principalmente $\mathrm{i}$ precari, in grande maggioranza giovani, senza peraltro che venisse adeguato il sistema di protezione sociale che tutela essenzialmente i lavoratori a tempo indeterminato. Se si aggiunge il fatto che l'enorme debito pubblico italiano graverà sulle giovani generazioni e che esse non hanno alcuna possibilità di migliorare la propria posizione occupazionale rispetto a quella dei genitori, si può facilmente concludere come i giovani di oggi si trovino di fronte a una situazione nuova rispetto alle giovani generazioni del Novecento: vale a dire il venir meno della prospettiva di un futuro migliore.

Un ulteriore elemento di novità nella storia recente è la crescente presenza sociale della popolazione anziana, alla quale è dedicato il volume di Antonio Golini e Alessandro Rosina (a cura di), Il secolo degli anziani. Come cambierà l'Italia, Bologna, Il Mulino, 2011. L'Italia è all'avanguardia di una transizione demografica che investirà tutto l'occidente e nella quale gli anziani andranno assumendo un peso viepiù rilevante. I pro- 
cessi migratori potranno soltanto attenuare gli effetti dell'incremento del rapporto tra popolazione anziana e popolazione attiva che rischia di porre problemi insormontabili alla finanza pubblica. Per ricostruire l'equilibrio del sistema occorrerà agire lungo due direzioni: trattenere più a lungo la popolazione al lavoro e inserire più rapidamente i giovani nel mercato del lavoro. È importante che entrambi questi processi abbiano luogo, nonostante vi siano evidenti elementi di contraddizione tra di essi, se si vuole scongiurare un drammatico impoverimento della nostra società; se nella prima direzione si sono fatti dei passi avanti, del tutto in controtendenza rispetto a quanto sarebbe necessario appare invece il ritardato ingresso dei giovani nel mercato del lavoro. Da ciò discende la centralità, per il futuro della nostra società, delle politiche del lavoro che dovrebbero essere prioritarie rispetto a qualsiasi altra preoccupazione di politica economica.

Se questa è la principale questione che pone questa transizione demografica, nondimeno l'aumento della popolazione anziana pone ulteriori sfide al sistema del welfare. È infatti inevitabile che la richiesta di assistenza per persone non autosufficienti sia destinata ad aumentare e che lo Stato sociale debba farsi parzialmente carico di questo problema che al momento grava pressoché interamente sulle famiglie, in particolare sulle figlie adulte degli anziani non autosufficienti che, quando attive sul mercato del lavoro, spesso ricorrono alle donne immigrate per gestire le esigenze di assistenza domestica. Sul piano delle politiche urbane, poi, va ripensata la pianificazione alla luce della crescente presenza di anziani, mentre sarà al contempo importante mettere a frutto la loro presenza ripensando la funzione che gli anziani possono avere nella nostra società e che informalmente già esercitano come prestatori di servizi alle famiglie o redistributori di risorse. È infatti oltre modo evidente che in Italia l'informale e privata redistribuzione delle risorse tra le generazioni sta compensando i limiti di iniquità generazionale del nostro welfare. Dato che ci troviamo di fronte da una parte a una popolazione anziana certamente più benestante che in passato, grazie al patrimonio cumulato con una 
vita di lavoro e a un welfare particolarmente generoso verso il sistema pensionistico, e dall'altra a una popolazione giovane impoverita e con scarse opportunità di miglioramento delle proprie condizioni, è naturale che all'interno delle strutture familiari si operino delle compensazioni.

Va comunque fatta un'importante precisazione metodologica che può essere utile a chi voglia cimentarsi con lo studio storico di questa categoria sociale che in Italia è ancora tutto da fare. Anche la categoria di anziano, non diversamente da quella di giovane, cambia insieme alla società. Nel 1951 gli over 65 erano 4 milioni, nel 2001 oltre 10 milioni, si prevede che possano essere 20 milioni nel 2050. Tuttavia, il prolungamento dell'aspettativa di vita pone forti dubbi alla comparabilità sociale di questi dati demografici. Coloro che avevano 65 anni nel 1951 potevano mediamente aspettarsi di vivere altri 13 anni; se mantenessimo gli ultimi 13 anni di vita come parametro dell'ingresso nella fase anziana, ecco che allora nel 1981 esso si sposterebbe da 65 a 70 anni e oggi a 75. Una nozione dinamica della definizione di anziano consente perciò di interpretare meglio i processi sociali e di orientare le politiche pubbliche in modo nuovo, come è accaduto con l'età pensionabile che è stata dinamicamente collegata alle aspettative di vita per conseguire un maggiore equilibrio nel rapporto tra contribuzione e spesa pensionistica.

Il pamphlet di Elisabetta Ambrosi e Alessandro Rosina, Non è un paese per giovani. L'anomalia italiana: una generazione senza voce, Venezia, Marsilio, 2009 entra nel pieno del dibattito pubblico sulle iniquità generazionali con una finalità interpretativa e polemica più che di resoconto scientifico. Va detto anzitutto che gli autori ritengono che questa iniquità sia il frutto della complicità di due generazioni: quella anziana abbarbicata nei posti di potere che non sembra avere alcuna intenzione di cedere, quella più giovane che pare subire l'ingiustizia generazionale senza reagire, accettando piuttosto la logica della cooptazione che premia alcuni perpetuando uno stato di subalternità.

I giovani ai quali si fa riferimento sono i trentenni - nati tra fine anni 
Sessanta e fine anni Settanta - (il libro è del 2009), una generazione che gli autori definiscono in termini strettamente anagrafici, dato che essa non si è messa in evidenza con particolari iniziative storicamente rilevanti. Questa generazione mostra scarsa combattività e presenza pubblica non solo per la sua marginalità demografica, ma anche perché si è trovata a subire il trauma della brusca interruzione di un'adolescenza dorata negli anni Novanta, quando si sono manifestati gli effetti della globalizzazione dei mercati e si è reso improcrastinabile fare i conti con un debito pubblico fuori controllo. L'ingresso nel mondo del lavoro di questa generazione è avvenuto all'insegna della deregulation del mercato che si è tradotta in un processo di precarizzazione che ridimensiona significativamente il reddito e rende meno autonomi e più ricattabili. Ciò spinge molti giovani a ricorrere alle famiglie di origine per compensare le difficoltà a sopravvivere sul mercato rinunciando a quella autonomia personale che costituisce un presupposto basilare della combattività sociale. Le difficoltà incontrate nella sfera pubblica generano poi facilmente la ricerca di vie d'uscita private, spesso caricando la sfera affettiva del compito di risarcire le frustrazioni pubbliche facendone l'unico riferimento della propria identità, esponendola così al rischio di rovinosi fallimenti.

Che vi sia un'acuta iniquità nel rapporto tra le generazioni è un dato di fatto incontrovertibile: nel 1965 un cinquantenne percepiva mediamente un salario più alto del $15 \%$ rispetto a un trentenne, oggi la differenza è diventata il 44\%, nonostante i giovani di oggi siano più istruiti e qualificati; un tempo le opportunità di carriera erano notevoli, oggi sono assai improbabili; le generazioni precedenti sono cresciute, oltre che con le opportunità professionali del boom economico, anche con la protezione di un welfare che oggi attraversa una crisi profonda e strutturale; ancora all'inizio degli anni Ottanta l'età media dei rappresentanti politici e sindacali era di 45 anni, oggi di 59; chi è entrato nel mercato del lavoro negli anni Novanta è destinato a percepire una pensione notevolmente più bassa di chi ci è entrato in precedenza, pur a fronte di un prolungamento 
degli anni di contribuzione che dovrà prestare; il debito che grava sulle giovani generazioni non ha paragone negli altri paesi europei.

Nonostante ciò, nel dibattito pubblico italiano è ricorrente una retorica giovanilistica e un'insistenza sul merito come strumento di selezione della classe dirigente che non può che suonare come vuota ipocrisia di fronte a una realtà sociale nella quale sembra inverarsi esattamente l'opposto. Gli autori sembrano riporre qualche maggiore speranza nella giovanissima generazione $-\mathrm{i}$ ventenni di oggi - che sta dando maggiori segni di orgoglio generazionale; tuttavia ritengono che siano necessarie alcune misure affinché si determini un'inversione di tendenza nei rapporti tra le generazioni: la riduzione del debito pubblico; una maggiore equità generazionale nella spesa sociale; il ringiovanimento delle cariche e il rinnovo della classe dirigente.

$\mathrm{Si}$ tratta di misure largamente condivise, e condivisibili, che sono diventate moneta corrente nel dibattito pubblico, tuttavia c'è da chiedersi se esse vadano davvero ad incidere nel profondo della disuguaglianza sociale che sta crescendo nella nostra società. Se infatti l'analisi della società in termini generazionali porta uno sguardo originale e indispensabile, come si è visto fin qui, non si può tuttavia evitare di metterne in evidenza anche i limiti. Di fronte alla profonda crisi che è precipitata negli ultimi anni, vi sono infatti chiari indizi di una reazione familista e classista che sembra attraversare le generazioni e fondarsi su altre forme di appartenenza. Gli stessi soggetti sociali strutturano il loro agire sulla base di un'identificazione di sé che non ha la generazione come principio fondamentale, quanto piuttosto delle strategie familiari, locali, di ceto, o di casta, come si preferisce nella vulgata giornalistica, volte a difendere il controllo di risorse economiche e simboliche che definiscono le gerarchie della società. In una fase di crisi, nella quale la prospettiva ritenuta più probabile è quella di un progressivo impoverimento, coloro che si trovano nella condizione di poter disporre di patrimonio e risorse agiscono al fine di conservare le posizioni acquisite per sé e per la ristretta cerchia sociale sulla quale si sa di poter contare e che rappresenta 
perciò essa stessa una risorsa. Si potrebbero citare numerosi esempi dal nostro presente che sono spie di questa tendenza: i ripetuti tentativi di alleggerire le tasse sulle proprietà immobiliari, a fronte di una tassazione sul lavoro che è la più alta d'Europa, segnala la pertinace difesa del patrimonio familiare, grande o piccolo che sia, che viene agitato come un baluardo inviolabile piuttosto che come l'oggetto di una politica fiscale; l'assenza di una strategia di finanza pubblica volta a sostenere la domanda di lavoro; la svalutazione dell'importanza del titolo di studio per l'accesso al mercato del lavoro e al contrario la rivalutazione della classe sociale di provenienza nella determinazione del destino sociale di un giovane mostrano infine quanto la posizione di partenza familiare, il reddito, il patrimonio, le relazioni, stiano tornando ad essere decisivi nella strutturazione della nostra società.

Nella seconda metà del Novecento, sindacati e partiti di massa hanno avuto una funzione decisiva nel promuovere la democratizzazione della società e il consolidamento della cittadinanza. Con la loro irreversibile crisi sul finire del secolo hanno lasciato un vuoto che è stato riempito dall'euforia neoliberale che sembrava poter (ri)proporre un modello di società imperniato sui pilastri fondamentali della proprietà privata e del profitto e affrancato dal potere arbitrario dei grandi apparati della rappresentanza sociale. Si è trattato di un ampio mutamento di paradigma che ha potuto prosperare in contesti politici molto diversi, dal Cile di Pinochet all'Inghilterra thatcheriana [Harvey 2007], che si è insediato in organi importanti della governance globale e che ha finito col riplasmare la stessa cultura politica di molte famiglie della sinistra europea, soprattutto dopo il 1989. È un modello che è stato sottoposto a critica fin dalla sua nascita, ma che ha mantenuto una sostanziale egemonia fino alla crisi apertasi nel 2007. Da quel momento in avanti sembra essersi aperta una fase nuova.

Occorrerà del tempo per affinare strumenti di analisi della fase storica che stiamo vivendo, quella della "società del declino e della crisi duratura", che richiede categorie tutt'affatto nuove rispetto a quelle con le 
quali si è interpretata e analizzata la società industriale dei trenta gloriosi e quella del "dopo-boom” [Doering-Manteuffel und Raphael 2010]. L'importante è che non si cerchino scorciatoie e di fronte a strategie familiari, a chiusure di casta, a ritorni di concezioni patrimonialiste e pratiche clientelari non si invochino presunti vizi italici codificati in qualche profonda struttura antropologica, come il familismo amorale e simili. Dalla rivoluzione neoliberista globale, alle politiche europee del dopo ' 89 , al modo in cui tutto ciò ha trovato traduzione nel nostro paese, sono stati intrapresi dei precisi indirizzi politici che hanno modificato sistemi valoriali, modalità di organizzazione economica e sociale, forme della partecipazione e della rappresentanza politica. È a partire dalla riconsiderazione critica di questi passaggi che possiamo sperare di comprendere qualcosa in più del nostro presente.

\section{Bibliografia}

Ambrosi E., Rosina A. 2009, Non è un paese per giovani. L'anomalia italiana: una generazione senza voce, Venezia: Marsilio.

Capuzzo P. 2012, Youth and Consumption, in F. Trentmann (ed.) 2012, The Oxford Handbook of the History of Consumption, Oxford: Oxford University Press, 601-617.

Doering-Manteuffel A., Raphael L. 2010, Nach Dem Boom: Perspektiven Auf Die Zeitgeschichte Seit 1970, Göttingen: Vandenhoeck \& Ruprecht.

Golini A., Rosina A. (eds.) 2011, Il secolo degli anziani. Come cambierà l’Italia, Bologna: Il Mulino.

Harvey D. 2007, Breve storia del neoliberismo, Milano: Il Saggiatore.

Mannheim K. 2008, Le generazioni, Bologna: Il Mulino, (I ed. 1928).

Schizzerotto A., Ugo Trivellato U., Sartor N. (eds.) 2011, Generazioni disuguali. Le condizioni di vita dei giovani di ieri e di oggi: un confronto, Bologna: Il Mulino. 